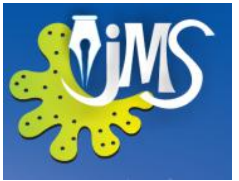

\title{
Numerical Implementation of Fourier Transforms and Associated Problems
}

\author{
Dhammika A. Tantrigoda $^{1}$ and Denushia S. Rodrigo ${ }^{2}$ \\ 1Department of Physics, University of Sri Jayewardenepura and \\ 2Department of Mathematics, University of Sri Jayewardenepura
}

\begin{abstract}
Fourier transforms initially used for the solution of problems in mathematical physics has today become a powerful tool of data analysis in wide spectrum of disciplines ranging from electrical engineering to social sciences. Its widespread applications can be attributed to the development of discrete Fourier transforms in middle part of the last century and subsequent development of fast Fourier transform algorithms which made its numerical implementation possible using digital computers. This paper reviews the limitations of the Fourier transform technique and associated problems and provide suggestions to overcome them.
\end{abstract}

KEYWORDS: Discrete Fourier transforms, Aliasing, High Frequency Instabilities, Truncation Errors, Symmetry Relationships 


\section{INTRODUCTION}

Physical quantities, either continues or sampled, that display spatial or temporal variations are generally referred to as signals. Discipline that deals with the acquisition and analysis of signals together with various operations carried out on them is known as signal processing. Some of the operations that signals are often subjected to are sampling, noise reduction, and feature extraction. Signal processing which was first developed as a branch of mathematics now has a wide spectrum of applications in disciplines such as electrical engineering, telecommunication, geophysics, meteorology, biological and medical sciences and even in social sciences.

One of the most powerful and time tested tools of signal processing is the Fourier transform method which includes Fourier analysis and synthesis. Famous French mathematician Jean-Baptiste Joseph Fourier in 1822 provided an elegant solution to the heat conduction equation for the first time in history making use of orthogonal properties of sine and cosine trigonometric functions. This method which originally introduced as a strategy of solving second order partial differential equations soon became useful tool of analyzing signals. Its importance was enhanced by several orders with the advent of discrete Fourier transforms during the middle part of the last century enabling the use of digital computers for signal processing (Bracewell, 1965; Brigham, 1974).

Fourier analysis allows decomposition of a signal into a large number of sinusoids with frequencies that are integer multiples of a fundamental frequency. Therefore this technique is widely used for the purpose of identifying periodicities in data. It is well known that the knowledge of periodicities in data is useful in making decisions in disciplines such as epidemiology (Zhang et al.,2014), climatology (Duchon and Hale, 2011), economics (Cherubini et al., 2009), medicine (Tomkins, 2000) and in environmental sciences.

Initially this technique was widely used by engineers, physicists and others who are having a strong background in mathematics. However, today due to the availability of computer packages that carry out the task of performing Fourier analysis, number of workers using this technique has increased by several folds. Some of them, especially those who are coming from non-mathematical backgrounds, use this technique without realizing its limitations and related problems. Application of this method without understanding its limitations may lead to erroneous results which are very often mathematical artifacts rather the solution to the problem that we are interested in. Therefore it is extremely important for those who are using this technique to have a good understanding of these limitations and problems. This paper reviews these limitations and related problems and provides suggestions on how to overcome them. Limitations that are being discussed here can be found in many text books which are generally meant for those who are having a strong background in mathematics. In this paper these limitations are presented and discussed so that users from non-mathematical backgrounds can easily be understood. 


\section{FoURIER TRANSFORMS, DisCRETE FOURIER TRANSFORMS AND FAST FOURIER TRANSFORM ALGORITHMS}

\subsection{Fourier Transforms:}

As mentioned earlier, the Fourier transform is a major tool that has numerous applications in the field of signal processing. This is also used in many other fields of science as a mathematical tool to transform a waveform or a function in time (or spatial domain) to a function in frequency domain (or wave number domain). The frequency domain function comprises an infinite number of sine and cosine terms. Fourier transform of a spatial domain continuous function $f(x)$ which is bounded and piecewise continuous is defined as,

$$
F(f)=\int_{-\infty}^{\infty} f(x) e^{-2 \pi i f x} d x
$$

Inverse Fourier transform is defined as,

$$
f(x)=\int_{-\infty}^{\infty} F(f) e^{2 \pi i f x} d f
$$

The frequency $f$ in the above expressions can be replaced by the wave number $k$ (where,

$$
\left.f=\frac{k}{2 \pi}\right) \text {. }
$$

It is clear from the above expression for the Fourier transform that it is a complex function in general and its real part gives the amplitudes of its cosine components while the imaginary part gives the amplitudes of its sine components. It can be easily understood that real part of the Fourier transform of a real function is a symmetric function while that of the imaginary part is an anti-symmetric function.

\subsection{Discrete Fourier transforms:}

The continuous Fourier transform converts a spatial or time domain signal of infinite duration into a continuous spectrum composed of an infinite number of sinusoids. In real world problems we always deal with finite duration signals that are discretely sampled. For such situations the Fourier transform definition has to be suitably modified. A Fourier transform modified to suit discrete functions are called a discrete Fourier transform (DFT) and relevant definitions and expressions are given below (Bracewell, 1965; Brigham, 1974; Press et al. 1992 and Terrell and Shark, 1996).

Let us consider a spatial domain function sampled at $N$ points $x_{\mathrm{j}}$ (where, $\mathrm{j}=0,1, . ., \mathrm{N}-1)$. Fourier transform and the Inverse Fourier transform of the sampled function are defined as

$$
X_{k}=\sum_{j=0}^{N-1} x_{j} e^{-2 \pi i j k / N}
$$

and

$$
x_{j}=\frac{1}{N} \sum_{k=0}^{N-1} X_{k} e^{2 \pi i j k / N}
$$

The above expression contains the most common normalization factor, $1 / N$. However, in different computational packages different normalization factors have been used. For example, the Mathematica Computer Algebra package uses $1 / \sqrt{N}$ as the normalization factor.

\subsection{Fast Fourier Transform Algorithm:}

The Fast Fourier transform (FFT) is an efficient algorithm to compute the discrete Fourier transform (DFT) and its inverse. It is an 
important algorithm for solving a large class of physical problems such as those in image processing, seismology and potential field studies in geophysics and market trend analysis in financial mathematics. A DFT transforms a data vector comprising a sequence of data into components of different frequencies. Computing it directly from the definition is often far too slow to be practical, especially when the vector has a large number of data values. The FFT is only a way to compute the same result more rapidly. Computing a DFT of a vector of $\mathrm{N}$ points, using the definition given above, requires $\mathrm{O}\left(\mathrm{N}^{2}\right)$ arithmetical operations, while an FFT can compute the same result only in $\mathrm{O}\left(\mathrm{N} \log _{2} \mathrm{~N}\right)$ operations. The Cooley and Tukey algorithm (1964) is the first ever FFT algorithm that can be seen in the published literature. This algorithm requires $\mathrm{N}$ to be a number that can be expressed as a power of 2 . It re-expresses a discrete Fourier transform of a vector of length $\mathrm{N}$ in terms of two smaller DFTs of lengths $\mathrm{N}_{1}$ and $\mathrm{N} 2$ (where, $\mathrm{N}=\mathrm{N}_{1} \mathrm{~N}_{2}$ ) and reduces the computation time to $\mathrm{O}\left(\mathrm{N} \log _{2}\right.$ $\mathrm{N})$. Some modern implementations (Press et al. 1992) of the FFT allow computation of FFT of a vector of any arbitrary length, not just those that are powers of two or the products of only small primes.

\section{Limitations and Practical Problems Related to the Fourier Transforms}

When a signal is studied by using numerical techniques, it is necessary to separate out the specific part of the signal that we are interested in (truncation of the signal) and sample it in an appropriate manner. Let $h(x)$ be a function of $x$ and assume it has been sampled evenly at intervals of $\Delta$. The sampled function can be expressed as, $h_{n}=h(n \Delta)$ where, $n=0,1,2,3, \ldots \ldots \ldots . N-1$

In the above expression $\Delta$ is the sampling interval and $n /(N \Delta)$, (where $n=-$ $N / 2, \ldots, N / 2)$ is referred to as frequency. The highest frequency $1 / 2 \Delta$ has a very special significance (which will be discussed later) and is known as the Nyquist frequency.

Both truncation and sampling reduce information contained in the signal. Hence in DFT we only estimate the Fourier transform of the function from a finite number of its sampled points. Truncation and sampling cause loosing of information as mentioned earlier creating problems such as aliasing, truncation error, Gibb's phenomenon and high frequency instabilities.

\subsection{Sampling Theorem and Aliasing:}

The success of the branch of knowledge that we referred to as digital signal processing completely depends on an important theorem in mathematics called sampling theorem. The sampling theorem states that a band limited signal can be completely recovered from its samples provided it is sampled at a rate at least equal to twice the Nyquist frequency $\left(f_{\mathrm{c}}\right)$.

If the signal contains frequencies higher than the Nyquist frequency, then the effect of the presence of such frequencies is reflected in the lower frequencies (spectral densities of higher frequencies are reflected in those of the lower frequencies) and this phenomenon is known as the aliasing effect. Press et al. (1992) illustrated this phenomenon for a time domain function and it is reproduced here for a spatial domain function with appropriate changes (Figure 1). 


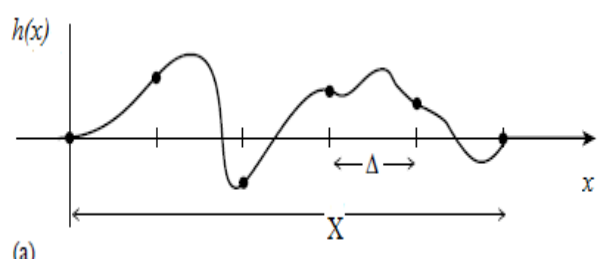

(a)

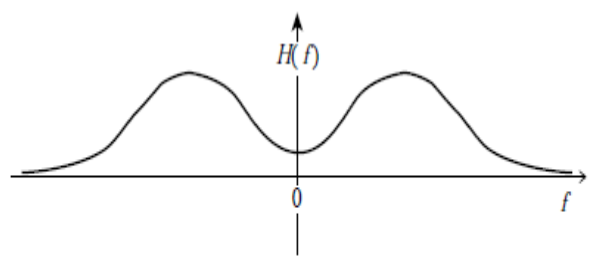

(b)

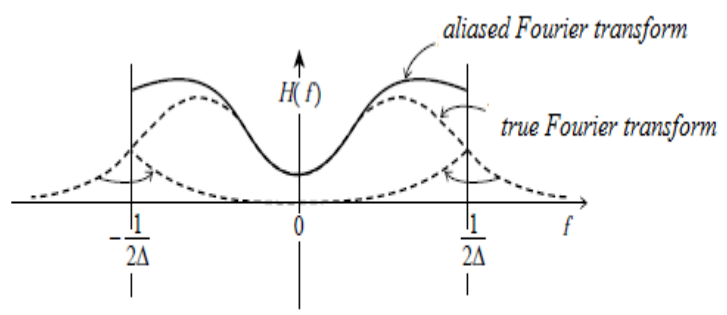

(c)

Figure 1: Illustration of aliasing due insufficient sampling. The continuous function shown in (a) is non zero only for a finite interval of length X. It follows that its Fourier Transform, whose modulus is shown schematically in (b), is not band limited but has finite amplitudes for all frequencies. If the original function is sampled with a sampling interval $\Delta$, as in(a), then the Fourier Transform (c) is defined only between plus and minus of the Nyquist critical frequency. Power outside that range is folded over or "aliased" into the range $(0-1 / 2 \Delta)$. This effect can be eliminated only by low pass filtering the original function before sampling.

\subsection{High Frequency Instabilities and Filtering:}

Almost all signals that we come across in the real world, either in time domain or in spatial domain, are contaminated with noise. These may be stemming from a variety of sources. Errors generated by inherent limitations of the measuring instrument and human errors are two obvious sources of noise. In addition to the above sources, there can be other sources of noise that are specific to the nature of the signal that we processing. To illustrate this let us consider a gravity or magnetic anomaly (signal) caused by a deep seated structure in the crust of the Earth. If we are interested in interpreting this anomaly, effect due to all the other structures are considered as noise. For example anomalies due to near surface narrow intrusive bodies are considered as noise as such anomalies will obliterate the anomaly or the signal that we are interested in. Most of the noise associated with a signal has short wavelengths or high frequencies. When a signal is subjected to frequency domain manipulations such as downward continuation, psudogravimetric transformation (Bott and Tantrigoda, 1986; Tantrigoda, 1982) or modeling in terms of a subsurface body (Parker, 1973; Rodrigo, 2012), there will be an enhancement of high frequency components including the noise component. Sometimes the amplification of the high frequency components is so pronounced and they deform the signal causing enormous problems in their analysis. This effect is known as high frequency instability and can be reduced to a reasonable level employing suitable filters. Tapering the frequency domain signal or application of cosine, Hanning or Hamming filters will reduce the high frequency instability to a reasonable level. 
The Cosine Taper window, $H(k)$, that is used to eliminate high frequency components is defined in the following manner:

$$
\begin{aligned}
& H(k) \\
& =\left\{\begin{array}{lr}
\frac{1}{2}\left(1+\cos \left[\frac{\pi\left(k-k_{l}\right)}{\left(k_{h}-k_{l}\right)}\right]\right) & k_{l} \geq k>k_{h} \\
1 & \text { otherwise }
\end{array}\right\}
\end{aligned}
$$

where, $k$ defines the range of the filter in the interval $\left[k_{l}, k_{h}\right]$ and wave numbers of the signal. The filter function is defined for both positive and negative frequencies. Illustration of cosine window is provided in the Figure 2.

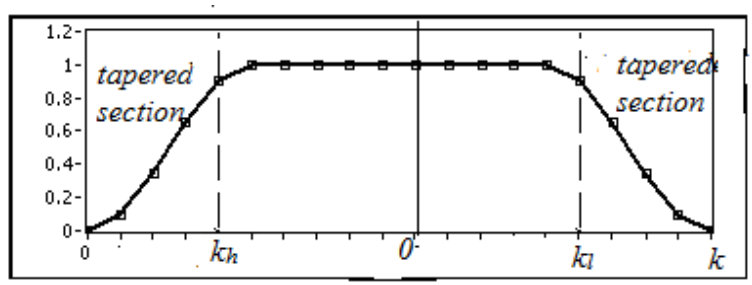

Figure 2: Cosine Taper window.

The Hanning filter is defined in the wave number domain as follows and it has been illustrated in Figure 3.

$$
H(k)=\left\{\begin{array}{lr}
\frac{1}{2}\left(1+\cos \left[\frac{\pi k}{k_{m}}\right]\right) & -k_{m} \leq k \leq k_{m} \\
0 & \text { otherwise }
\end{array}\right\}
$$

Where, $\mathrm{k}$ defines the range of the filter in the interval $[-\mathrm{km}, \mathrm{km}]$ and wave numbers of the signal. The window function is shown in Figure 3.

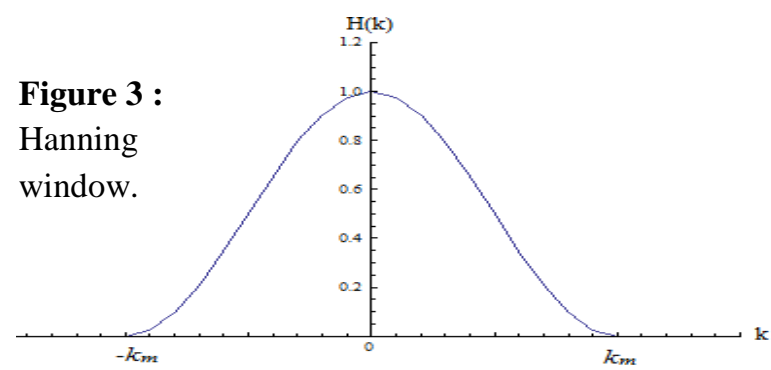

The other filter that is usually used for this purpose is the Hamming filter and its definition is given below. It has a shape that is closely similar to that of the Hanning filter.

$H(k)$

$=\left\{\begin{array}{cc}0.54+0.46 \cos \left[\frac{\pi k}{k_{m}}\right] & -k_{m} \leq k \leq k_{m} \\ 0 & \text { otherwise }\end{array}\right\}$

Where, the interval $[-\mathrm{km}, \mathrm{km}]$ and $\mathrm{k}$ define the range of the filter and wave numbers of the signal respectively.

\subsection{Truncation Error:}

Almost all real world signals are of long duration or of long length. It can even be of infinite duration or of length. Normally we are only interested in a limited portion of a signal and we have to extract that portion of the signal by truncating it. The mathematical equivalent of truncation of a signal is multiplication of the signal by a rectangular window of unit height or by a box-car function. According to the convolution theorem the Fourier transform of the truncated function is equivalent to convolution of the Fourier transform of the original signal and that of the box-car function. It is well known that the Fourier transform of a box-car function is a sine cardinal function or a "sinc" function, $\left(\frac{\sin f}{f}\right)$. The most prominent characteristic of the "sinc" function is its side lobes on either side of its principal maximum. These side lobes introduce spurious ripples at the high frequency end of the frequency domain function introducing undesirable deformation to the spatial domain function (Bracewll, 1965; Blackman and Tukey, 1959; Brigham, 1974 ). This effect is very often referred to as the truncation effect and has been illustrated in 
Figure 4. Employing suitable filters or simply by selecting a large portion of the signal, the truncation error can be minimized. When the length of the box function is increased its Fourier transform becomes closer to a delta function devoid of side lobes reducing "ripple effect" mentioned above.
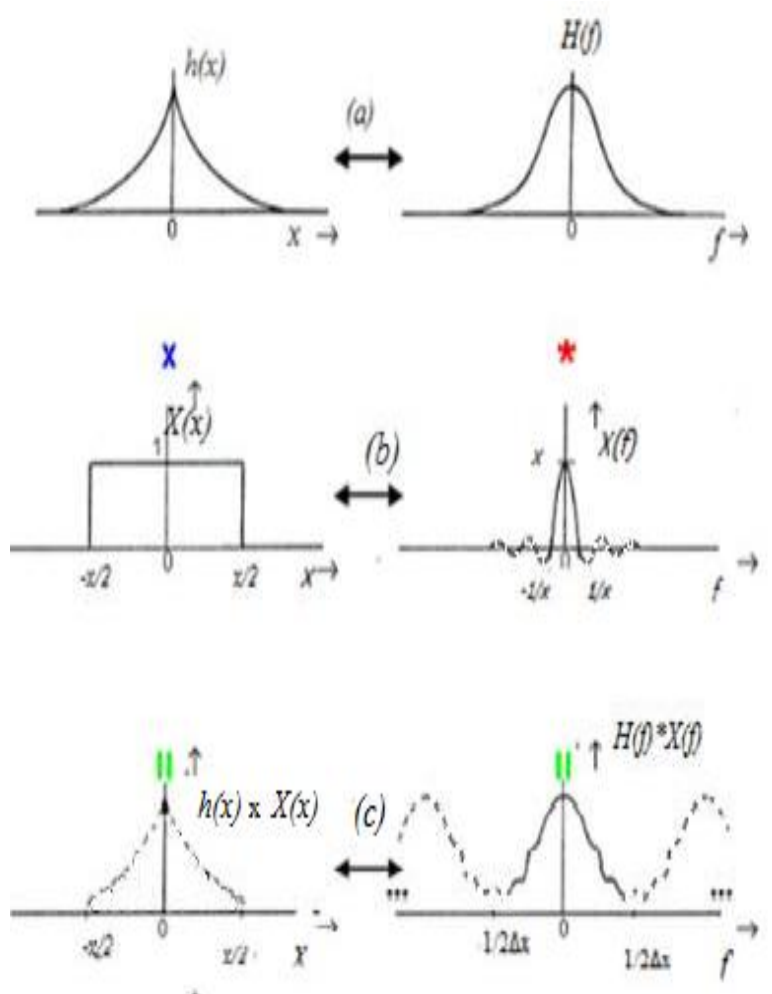

Figure 4: Instability due to truncation error. (a) :Spatial domain function and its frequency domain function, $(b)$ : Box car function and its Fourier transform (sinc function) with side lobs.(c):Truncated function $(h(x) \times X(x))$ and its Fourier transform $(H(f) * X(f))$.

\subsection{Gibb's Phenomenon:}

Sampling a signal with a certain frequency is equivalent to removing of the effects due to frequencies higher than that frequency. Since the frequency domain (sampled) function or the
Fourier transform does not contain all the information in the original function, it is not possible to recover the original spatial domain function from its Fourier transform. This is known as the Gibb's phenomenon. Bracewell (1965) has beautifully illustrated the Gibb's phenomenon considering a step function. The effect of the Gibb's phenomenon can never be removed from a signal as it is an inherent effect caused by sampling a signal. However, it can be reduced simply by increasing sampling frequency. The Gibb's phenomenon has been illustrated below using a square wave. Illustration of Gibb's phenomenon is shown in the Figure 5. Here, the square wave function is defined as,

$$
f(x)=\left\{\begin{array}{rc}
1 & 0 \leq x \leq \pi \\
-1 & -\pi \leq x<0
\end{array}\right.
$$

$f(x)$ is periodic beyond the above interval with a period $2 \pi$.

Fourier series of the function can be written as,

$$
f(x)=\frac{4}{\pi}\left[\frac{\sin x}{1}+\frac{\sin 3 x}{3}+\frac{\sin 5 x}{5}+\cdots+\frac{\sin (2 n-1) x}{(2 n-1)}+\cdots\right]
$$

Partial sums of the above series for $n=1, n=11, n=26$ and $n=41$ and are plotted with $\mathrm{x}$ below (Figure 5). As can be seen from this illustration when the number of terms considered is increased the shape of the figure comes closer to a square wave. At the same time we can observe that the frequency of "wiggles" in flat region of the figure increases instead of completely disappearing. It is obvious these will disappear only when infinite numbers of terms are added. Therefore it is impossible to regain the original function from a Fourier transform of a sampled function. 


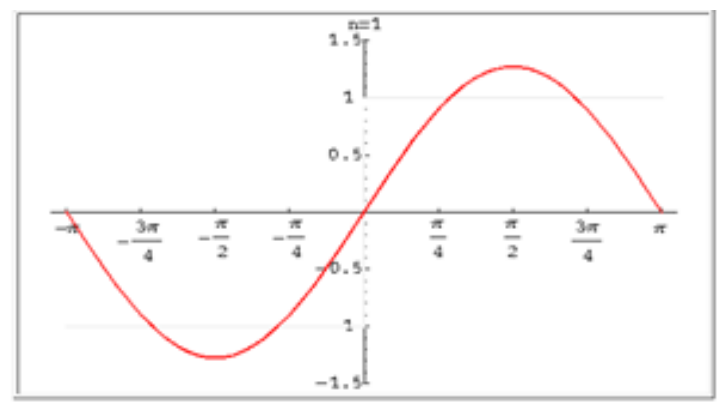

(A)

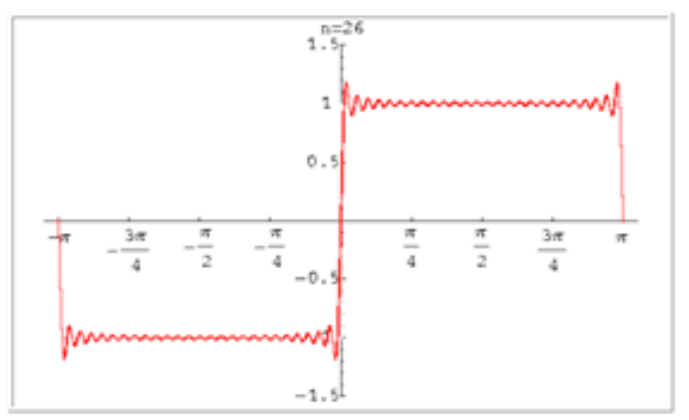

(C)

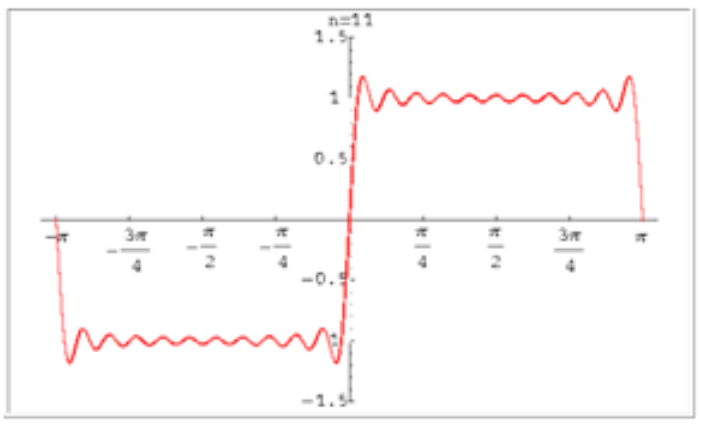

(B)

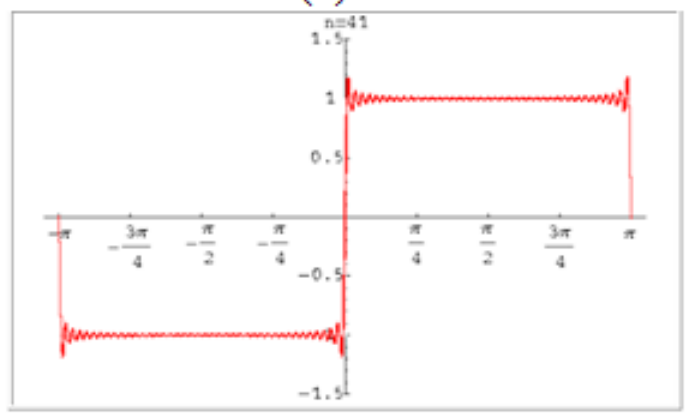

(D)
Figure 5: Illustration of Gibb's phenomenon using a square wave. The figure $\mathrm{A}$ gives the first term of the series and figures B, C and D give the partial sums of the 11,26 and 41 terms respectively. It can be observed that when the number of terms in the partial sum increases, the shape of the waveform becomes closer to a square wave, but the frequency of the wiggles in the waveform increases. Note only the region of the wave from - is depicted in the figure.

\section{Symmetries of Fourier transforms of real functions}

Fourier transform of a real function is a complex function with some special properties. It can be shown easily that the real part of a Fourier transform of real function is symmetric while its imaginary part is anti-symmetric (Bracewell, 1965). These symmetries for one dimensional and two dimensional functions are illustrated in the Figure 6 and in Figure 7.

Let us consider a spatial domain real function. As explained above the Fourier transform of this function has a symmetric real part and anti symmetric imaginary part. If we do some mathematical manipulations on the Fourier transform and if we expect the inverse Fourier transform of this function to be real in the spatial domain for some physical reason then we have to adjust the Fourier transform so that it has the above mentioned symmetries. Otherwise we will end up with an imaginary function which does not have any physical meaning. 


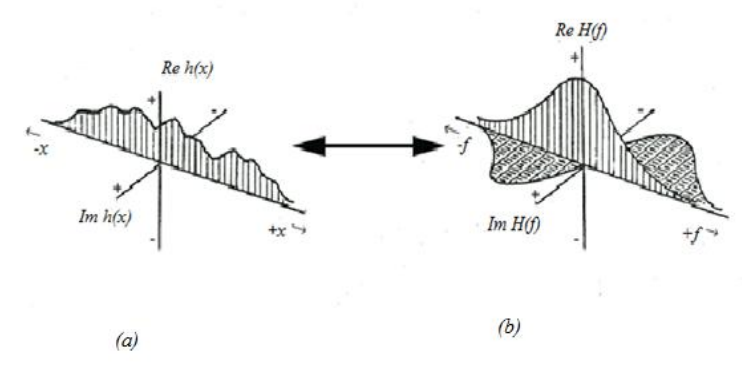

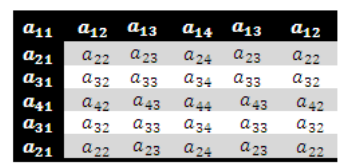

(a)

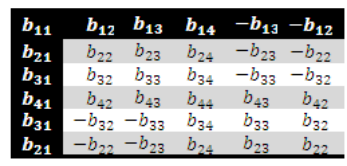

(c)

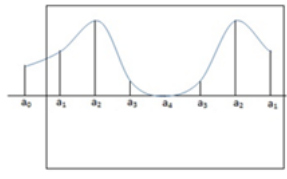

(b)

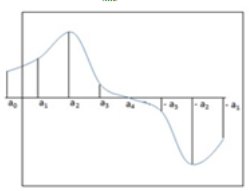

(d)
Figure 6: Symmetry relationships of real and imaginary parts of the Fourier transform of a real function $h(x)$. Spatial domain function is depicted Figure 6(a).Symmetric nature of the real part of the Fourier transform and the antisymmetric nature of the imaginary part of the Fourier transform have been illustrated in Figure 6(b).
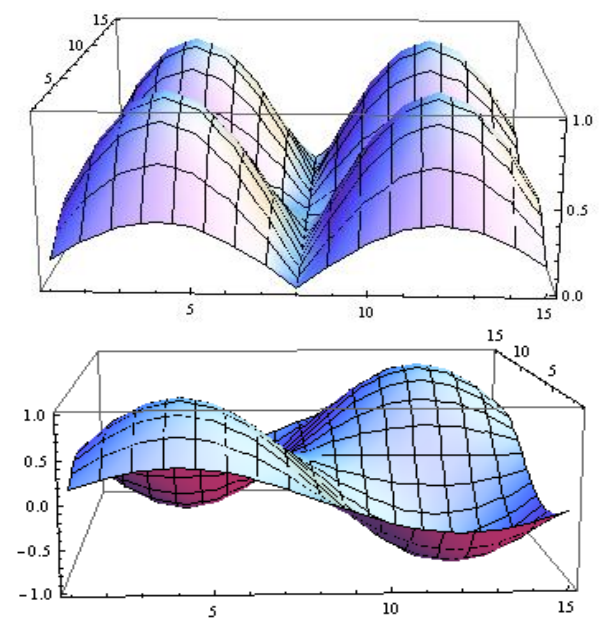

(e)
Figure 7: Illustration of symmetry relationships of Fourier transform of a $6 \times 6$ two dimensional real function (7.a and 7.b give real part and a cross-section while 7.c and 7.d give imaginary part and a cross-section). Note that first row of 7(a) (excluding $\mathrm{a}_{11}$ ) is symmetric around $a_{14}$ and similarly its first column is symmetric around $a_{41}$. Also note that first row of 7(c) is (excluding $\mathrm{b}_{11}$ ) anti-symmetric around $\mathrm{b}_{14}$ and similarly its first column is antisymmetric around $\mathrm{b}_{41}$. Relationships of remaining terms are self-evident and are also depicted in figure $7(e)$.

\section{Limitations of Fourier Transforms and Novel Trends in the Signal Processing}

The Fourier transform of a continuous signal is an expression that indicates how the amplitudes of various frequencies present in a signal varies with the frequency. In the case of continuous Fourier transforms we use the knowledge of the signal from $-\infty$ to $\infty$ and a s a result of this we are in a position to determine amplitudes of infinite number of frequencies or in more practical terms amplitudes of all frequencies present in the signal. When dealing with real world signals we always have to truncate it and sample it and this will limit the number of frequencies that we can extract from it. We can only determine the amplitudes of frequencies equal to the half the number of samples subjected to the limitations imposed by the sampling theorem. Further, we only obtain amplitudes of frequencies given by $n /(N \Delta)$, where $n=-N / 2, \ldots ., N / 2$ and $\Delta$ is the sampling interval. Another limitation of Fourier transforms is that it does not contain any local information. In order to address this problem windowed Fourier transforms or short interval Fourier transforms have been introduced. In 
windowed Fourier transforms, signal is divided into several intervals according to the local features present in the signal and the Fourier transform of each interval is taken separately. More elegant method of addressing this and several other problems in Fourier transform method has been first introduced by Morlet (1982) and later developed by many others (Grossman and Morlet, 1984; Daubechies, 1990). The wavelet transform has become a widely used technique in signal processing today. In spite all these new developments the Fourier transform is still used by many researchers through out of the world in a multitude of disciplines.

\section{REFERENCES}

BOTT, M.H.P. AND TANRIGODA, D.A. (1987). Interpretation of gravity and magnetic anomalies over the Mull Tertiary intrusive complex, NW Scotland, Journal of the Geological Society of London, Vol . 144, 17-28.

BRIGHAM, E.O. (1974). The Fast Fourier Transform, Prentice Hall Inc., New Jersey, 228 pp.

BRACEWELL, R.N. (1965). The Fourier Transform and its applications, McGraw Hill, London, 444 pp.

BLACKMANN, R.B. AND TUKEY, S.W., (1959). The measurement of power spectra from the point of view of communication engineering, Dover and New York, 190 pp.

CHERUBINI, U., LUNGA, G.D., MULINACCI, S. AND ROSSI, P., (2009). Fourier Transform Methods in Finance, Wiley, $256 \mathrm{pp}$.

COOLEY, J.W. AND TUKEY, J.W. (1965). An algorithm for machine calculation of of complex Fourier series. Math. Comp., 19, 297-301.
DUCHON, C. AND HALE, R., (2011). Time series analysis in Meteorology and Climatology: An Introduction, WileyBlackwell, 262 pp.

PRESS, W.H., TUEKOLSKY, S.A., VETTERLING,W.T., FLANNERY, B.P. (1992). Numerical Recipes in C: The Art of Scientific Computing, Cambridge University Press, 496 pp.

PARKER, R.L., (1973). The rapid calculation of potential field anomalies, Geophysical Journal of the Royal Astronomical. Society, 31, 447-455.

RODRIGO, D.S. (2014). Modeling of gravity anomalies using a Fourier iterative technique, MPhil Thesis (unpublished), Univ. of Sri Jayewardenepura.

TOMKINS, W.J. (2000). Biomedical digital signal processing, Prentice Hall of India, New Delhi, 368 pp.

TERRELL, T.J. AND SHARK, L. (1996). Digital signal processing: A student guide, MacMillan and Press, London, 326 pp.

TANTRIGODA, D.A. (1982). Interpretation of magnetic anomalies using the pseudogravimetric transformation and other methods with application to Tertiary intrusive complexes in NW Scotland, Ph.D. Thesis (unpublished), Univ. of Durham, UK.

ZHANG, T., YANG, M., XIAO, X., FENG, Z., LI, C., ZHOU, Z., REN, Q. AND LI, X. (2014). Spectral analysis based on fast Fourier transformation (FFT) of surveillance data: the case of scarlet fever in China, Epidemiology and Infection, Vol. 142(3), 520-529. 